\title{
AN ALTERNATIVE TO RIEMANN-SIEGEL TYPE FORMULAS
}

\author{
GHAITH A. HIARY
}

\begin{abstract}
Simple unsmoothed formulas to compute the Riemann zeta function, and Dirichlet $L$-functions to a power-full modulus, are derived by elementary means (Taylor expansions and the geometric series). The formulas enable square-root of the analytic conductor complexity, up to logarithmic loss, and have an explicit remainder term that is easy to control. The formula for zeta yields a convexity bound of the same strength as that from the Riemann-Siegel formula, up to a constant factor. Practical parameter choices are discussed.
\end{abstract}

\section{INTRODUCTION}

The Riemann zeta function is defined for $s=\sigma+i t$ by $\zeta(s)=\sum_{n=1}^{\infty} n^{-s}, \sigma>1$. It can be analytically continued everywhere except for a simple pole at $s=1$. The zeta function satisfies the functional equation $\zeta(s)=\chi(s) \zeta(1-s)$ where $\chi(s):=$ $\pi^{s-1 / 2} \Gamma((1-s) / 2) / \Gamma(s / 2)$. One is usually interested in numerically evaluating $\zeta(\sigma+i t)$ on the critical line $\sigma=1 / 2$ (e.g. to verify the Riemann hypothesis). However, one cannot use the Dirichlet series $\sum_{n=1}^{\infty} n^{-s}$ to numerically evaluate zeta when $\sigma<1$ because the series diverges. Rather, one can use partial summation and integration by parts to analytically continue the series to $\sigma>0$, obtaining

$$
\zeta(s)=\sum_{1 \leq n<M} \frac{1}{n^{s}}+\frac{M^{-s}}{2}+\frac{M^{1-s}}{s-1}+\mathcal{R}_{M}(s), \quad\left|\mathcal{R}_{M}(s)\right| \leq \frac{\mathfrak{q}(s)}{\sigma M^{\sigma}},
$$

where $\mathfrak{q}(s):=|s|+3$ is the analytic conductor of zeta; see [11. The analytic conductor terminology was introduced by Iwaniec and Sarnak; see 10] for example. This terminology will be useful when we generalize our formulas to Dirichlet $L$ functions, and it ensures that $\log \mathfrak{q}(s)>0$. We remark, though, that the precise definition of the analytic conductor does not affect the asymptotic content of the results, since $\mathfrak{q}(s)$ needs only be of a comparable size to $|s|$.

Formula (11) can be viewed as consisting of a main sum $\sum_{n<M} n^{-s}$, an extra term $M^{-s} / 2+M^{1-s} /(s-1)$, and a remainder $\mathcal{R}_{M}(s)$. The main sum accounts for the bulk of the computational effort, the extra term can be computed easily, and the remainder can be controlled by choosing $M$ accordingly. For example, one can ensure that $\left|\mathcal{R}_{M}(s)\right|<\epsilon$ on taking $M>(\mathfrak{q}(s) /(\sigma \epsilon))^{1 / \sigma}$. So when $\sigma=1 / 2$, the main sum consists of $\gg \mathfrak{q}(s)^{2}$ terms, even if $\epsilon=1$ say. Using a more careful analysis, however, one can show that $\mathcal{R}_{M}(s) \ll M^{-\sigma}$ if $M \gg \mathfrak{q}(s)$. Alternatively, one can use the Euler-Maclaurin summation (see 92 ) which allows for far more accuracy. In either case, though, the resulting main sum is of length $\gtrsim \mathfrak{q}(s)$. So

2010 Mathematics Subject Classification. Primary 11M06, 11Y16; Secondary 68Q25.

Key words and phrases. Riemann zeta function, Dirichlet $L$-functions, algorithms.

Preparation of this material is partially supported by the National Science Foundation under agreement No. DMS-0932078 (while at MSRI) and DMS-1406190, and by the Leverhulme Trust (while at the University of Bristol). 
these formulas are rather impractical for numerical computations on single processor when $t \gtrsim 10^{10}$, say, especially if high precision is sought. This is unfortunate since they are simple to derive and analyze, and have explicit error bounds. So, instead, one typically uses the Riemann-Siegel asymptotic formula which has a much shorter main sum of length $\lfloor\sqrt{t /(2 \pi)}\rfloor$ (see \$2). The Riemann-Siegel formula was discovered around 1932 in Riemann's unpublished papers by C.L. Siegel. Some of its history is narrated in [6, Chapter 7]. In lieu of the Riemann-Siegel formula, one can use the efficient smoothed formulas in 14 .

We propose a new method for computing zeta based on slowly converging Dirichlet series such as (1). Then we generalize our method to Dirichlet $L$-functions to a power-full modulus. Interestingly, our results can be derived without knowing about the functional equation of the associated $L$-function, nor using analysis of similar strength, such as the Poisson summation. To state the results, we introduce some notation. Let

$$
f_{s}(z):=\frac{e^{s z}}{(1+z)^{s}}, \quad f_{s}(0)=1, \quad g_{K}(z):=\sum_{k=0}^{K-1} e^{k z}=\frac{e^{K z}-1}{e^{z}-1}, z \notin 2 \pi i \mathbb{Z},
$$

where $f_{s}^{(j)}(z)$ and $g_{K}^{(j)}(z)$ denote the $j$-th derivative in $z$. We choose integers $u_{0} \geq 1$, $v_{0} \geq u_{0}$, and $M \geq v_{0}$, and construct sequences $K_{r}=\left\lceil v_{r} / u_{0}\right\rceil$ and $v_{r+1}=v_{r}+K_{r}$ for $0 \leq r<R$, where $R:=R\left(v_{0}, u_{0}, M\right)$ is the largest integer such that $v_{R}<M$. We define $K_{R}:=\min \left\{\left\lceil v_{R} / u_{0}\right\rceil, M-v_{R}\right\}$, so that $v_{R+1}=M$. Then we divide the main sum in (11) into an initial sum of length $v_{0}$, followed by $R+1$ consecutive blocks where the $r$-th block starts at $v_{r}$ and has length $K_{r}$. The sequences $K_{r}$ and $v_{r}$ are so defined in order to implement a more efficient version of dyadic subdivision of the main sum. There will be substantial flexibility in choosing them (need only $K_{r}-1 \leq v_{r} / u_{0}, u_{0} \geq \sqrt{\mathfrak{q}(s)}$ ), but we do not exploit this here. We plan to approximate the $r$-th block $\sum_{v_{r} \leq n<v_{r}+K_{r}} n^{-s}$ by $v_{r}^{-s} B_{r}(s, m)$ where

$$
B_{r}(s, m):=\sum_{j=0}^{m} \frac{f_{s}^{(j)}(0)}{j !}, \frac{g_{K_{r}}^{(j)}\left(-s / v_{r}\right)}{v_{r}^{j}},
$$

which is a linear combination of a geometric sum and its derivatives. Also, we let

$$
\begin{gathered}
\mathcal{B}_{M}\left(s, u_{0}, v_{0}\right):=\sum_{r=0}^{R} v_{r}^{-\sigma} \min \left\{g_{K_{r}}\left(-\sigma / v_{r}\right),\left|\csc \left(t /\left(2 v_{r}\right)\right)\right|\right\}, \\
\epsilon_{m}(s, u):= \begin{cases}\frac{3.5 e^{0.78(m+1)}}{(m+1)^{(m+1) / 2}} \frac{|s|^{(m+1) / 2}}{u^{m+1}}, & m \leq|s| / 4, \\
\frac{2^{m} e^{0.194|s|}}{u^{m}}, & m>|s| / 4 .\end{cases}
\end{gathered}
$$

We prove the following theorem in $\$ 3$.

Theorem 1.1. Given $s=\sigma+i t$ with $\sigma>0$, let $u_{0}$ and $v_{0}$ be any integers satisfying $v_{0} \geq u_{0} \geq 2 \max \{6, \sqrt{\mathfrak{q}(s)}, \sigma\}$. Then for any integers $M \geq v_{0}$ and $m \geq 0$ we have

$$
\zeta(s)=\sum_{n=1}^{v_{0}-1} \frac{1}{n^{s}}+\sum_{r=0}^{R} \frac{B_{r}(s, m)}{v_{r}^{s}}+\frac{M^{-s}}{2}+\frac{M^{1-s}}{s-1}+\mathcal{T}_{M, m}\left(s, u_{0}, v_{0}\right)+\mathcal{R}_{M}(s),
$$

where $\left|\mathcal{T}_{M, m}\left(s, u_{0}, v_{0}\right)\right| \leq \epsilon_{m}\left(s, u_{0}\right) \mathcal{B}_{M}\left(s, u_{0}, v_{0}\right)$. We have $R<2 u_{0} \log \left(M / v_{0}\right)+1$. 
We could have used the main sum from the Euler-Maclaurin formula, instead of the main sum in (1), to derive Theorem 1.1. This permits one to choose $M$ smaller. Indeed, replacing $\mathcal{R}_{M}(s)$ by the Euler-Maclaurin correction terms, one can restrict $M \ll \mathfrak{q}(s)$ while retaining high accuracy. In this case, Theorem 1.1. applied with $m=0$, leads to a simple proof of the bound $\zeta(1 / 2+i t) \ll \mathfrak{q}(1 / 2+i t)^{1 / 4}$; see corollary 5.2 in $\$ 5$. The truncation error $\mathcal{T}_{M, m}$ in Theorem 1.1 is bounded by $\epsilon_{m} \mathcal{B}_{M}$, where, by lemma 3.2, we have $\mathcal{B}_{M}\left(s, u_{0}, v_{0}\right) \leq v_{0}^{-\sigma}+\left(M^{1-\sigma}-v_{0}^{1-\sigma}\right)(1-\sigma)^{-1}$ if $\sigma \neq 1$, and $\mathcal{B}_{M}\left(s, u_{0}, v_{0}\right) \leq v_{0}^{-\sigma}+\log \left(M / v_{0}\right)$ if $\sigma=1$. This estimate is quite generous, however. It can be improved by computing $\mathcal{B}_{M}\left(s, u_{0}, v_{0}\right)$ directly, which should yield a bound like $u_{0} /\left(\sigma v_{0}^{\sigma}\right)$. The said computation can be done in about $R$ steps, and so it is subsumed by the computational effort for the main sum. In either case, the remainder term is clearly easy to control when $u_{0} \geq \sqrt{\mathfrak{q}(s)}$, due to the rapid decay of $\epsilon_{m}\left(s, u_{0}\right)$ with $m$ (decays like $\left.1 /\lfloor(m+1) / 2\rfloor !\right)$.

The main sum in Theorem 1.1 has $v_{0}+(m+1)(R+1)$ terms, where each term is, basically, a geometric sum. To ensure that $\left|\mathcal{T}_{M, m}(s)\right|+\left|\mathcal{R}_{M}(s)\right|<\epsilon$ for $\sigma=1 / 2$, it suffices to take $M \ll(\mathfrak{q}(s) / \epsilon)^{2}$ and $m \ll \log (\mathfrak{q}(s) / \epsilon)$. Since $R \leq 2 u_{0} \log \left(M / v_{0}\right)+1$, this is of length $\ll v_{0}+u_{0} \log ^{2}(\mathfrak{q}(s) / \epsilon)$ terms. Choosing $u_{0}=v_{0}=2\lceil\sqrt{\mathfrak{q}(s)}\rceil$, which is a typical choice, the main sum thus consists of $\ll \sqrt{\mathfrak{q}(s)} \log ^{2}(\mathfrak{q}(s) / \epsilon)$ terms. We show how to compute these terms (geometric sums) efficiently in $₫ 4$ using $\ll \log (\mathfrak{q}(s) / \epsilon)$ precision. So, put together, the complexity of the formula in Theorem 1.1 depends only logarithmically on $M$ and the error tolerance $\epsilon$. The formula enables square-root of the analytic conductor complexity, up to logarithmic loss, without using the functional equation, or the approximate functional equation. Also, the usual factor $\chi(s)$ does not appear, and the conditions on $v_{0}$ and $u_{0}$ imply that $v_{0} u_{0} \gg \mathfrak{q}(s)$. Nevertheless, the idea behind the theorem is fairly simple. Writing $n^{-s}=e^{-s \log n}$, we have $\sum_{v \leq n<v+K} n^{-s}=v^{-s} \sum_{0 \leq k<K} e^{-s \log (1+k / v)}$. So if $K / v \ll 1 / \sqrt{\mathfrak{q}(s)}$, as we will have, then $s \log (1+k / v)=s k / v+O(1)$. In particular, using Taylor expansions, we can approximate $\sum_{0 \leq k<K} e^{-s \log (1+k / v)}$ by a linear combination of the geometric sum $g_{K}(-s / v)$ and several of its derivatives. These geometric sums are easy to compute, which is the reason for the savings.

One can shorten the length of the main sum in Theorem 1.1 to be roughly $\mathfrak{q}(s)^{1 / 3}$. But then instead of obtaining linear exponential sums, one obtains quadratic exponential sums. The length can be further shortened, leading to cubic and higher degree exponential sums. In view of this, Theorem 1.1 belongs to the family of methods for computing zeta that were derived in 9 . And like these methods (see [8]), Theorem 1.1 can be generalized to Dirichlet $L$-functions $L(s, \chi), \chi \bmod q$, when $q$ is power-full. To this end, define the analytic conductor for $L(s, \chi)$ by $\mathfrak{q}(s, \chi):=q(|s|+3)$. If $\chi \bmod q$ is non-principal, then we have the trivial bound $\left|\sum_{n} \chi(n)\right|<q$. Combined with partial summation we obtain, for $\sigma>0$, that 1

$$
L(s, \chi)=\sum_{1 \leq n<M} \frac{\chi(n)}{n^{s}}+\mathcal{R}_{M}(s, \chi), \quad\left|\mathcal{R}_{M}(s, \chi)\right| \leq \frac{2 \mathfrak{q}(s, \chi)}{\sigma M^{\sigma}} .
$$

1 To estimate $\mathcal{R}_{M}(s, \chi)$, we used the following partial summation formula (see [14]): Let $f: \mathbb{Z}^{+} \rightarrow \mathbb{C}$ and $g: \mathbb{R} \rightarrow \mathbb{C}$ such that $g^{\prime}$ exists on $[1, x]$. Then for $y \in[1, x]$ we have

$$
\sum_{y<n \leq x} f(n) g(n)=\left(\sum_{y<n \leq x} f(n)\right) g(x)+\left(\sum_{1 \leq n \leq y} f(n)\right)(g(x)-g(y))-\int_{y}^{x}\left(\sum_{1 \leq n \leq \tau} f(n)\right) g^{\prime}(\tau) d \tau \text {. }
$$


We will only consider the case $q=p^{a}$ for $p$ prime. As in Theorem 1.1 we divide the main sum in (6) into an initial sum of length $v_{0}$, followed by $R+1$ consecutive blocks, where the $r$-th block starts at $v_{r}$ and has length $K_{r}$. Let $g_{K}(z, \chi, v):=$ $\sum_{0 \leq k<K} \chi(v+k) e^{k z}$. Then, in analogy with zeta, we approximate the $r$-th block $\sum_{v_{r} \leq n<v_{r}+K_{r}} \chi(n) n^{-s}$ by $v_{r}^{-s} B_{r}(s, \chi, m)$ where

$$
B_{r}(s, \chi, m):=\sum_{j=0}^{m} \frac{f_{s}^{(j)}(0)}{j !} \frac{g_{K_{r}}^{(j)}\left(-s / v_{r}, \chi, v_{r}\right)}{v_{r}^{j}},
$$

and $g_{K}^{(j)}(z, \chi, v)$ denotes the $j$-th derivative in $z$. The analogue of $\mathcal{B}_{M}$ from Theorem 1.1 is going to be more complicated to define. To this end, let $b:=\lceil a / 2\rceil$ and, for $0 \leq d<p^{b}$, let $H_{r, d}:=\left\lceil\left(K_{r}-d\right) / p^{b}\right\rceil$ and $w_{r, d}:=2 \pi \overline{v_{r}+d} L / p^{a-b}$ where $L$ is as in lemma 1.3 and $\left(\overline{v_{r}+d}\right)\left(v_{r}+d\right) \equiv 1 \bmod p^{a}$ if $\operatorname{gcd}\left(v_{r}+d, p\right)=1$. Then let

$$
\begin{array}{r}
\mathcal{B}_{M}\left(s, \chi, u_{0}, v_{0}\right):=\sum_{r=0}^{R} \sum_{d=0}^{p^{b}-1} \delta_{\operatorname{gcd}\left(v_{r}+d, p\right)=1} \min \left\{e^{-\sigma d / v_{r}} g_{H_{r, d}}\left(-p^{b} \sigma / v_{r}\right),\right. \\
\left.\left|\csc \left(w_{r, d} / 2-p^{b} t /\left(2 v_{r}\right)\right)\right|\right\} v_{r}^{-\sigma} .
\end{array}
$$

In $₫ 3$, we prove the following.

Theorem 1.2. Given $s=\sigma+i t$ with $\sigma>0$, a non-principal Dirichlet character $\chi \bmod p^{a}$ with $p$ a prime, let $b=\lceil a / 2\rceil$, and let $u_{0}$ and $v_{0}$ be any integers satisfying $v_{0} \geq u_{0} \geq 2 \max \{6, \sqrt{\mathfrak{q}(s)}, \sigma\}$. Then for any integers $M \geq v_{0}$ and $m \geq 0$ we have

$$
L(s, \chi)=\sum_{n=1}^{v_{0}-1} \frac{\chi(n)}{n^{s}}+\sum_{r=0}^{R} \frac{B_{r}(s, \chi, m)}{v_{r}^{s}}+\mathcal{T}_{M, m}(s, \chi)+\mathcal{R}_{M}(s, \chi),
$$

where $\left|\mathcal{T}_{M, m}(s, \chi)\right| \leq \epsilon_{m}\left(s, u_{0}\right) \mathcal{B}_{M}\left(s, \chi, u_{0}, v_{0}\right)$. We have $R<2 u_{0} \log \left(M / v_{0}\right)+1$.

We use the Postnikov character formula in $\$ 3$ to show that $g_{K}(z, \chi, v)$ can be written as a sum of $p^{b}$ geometric sums.

Lemma 1.3. Given a Dirichlet character $\chi \bmod p^{a}$ with $p$ a prime, let $b=\lceil a / 2\rceil$, and $H_{d}:=\left\lceil(K-d) / p^{b}\right\rceil$. Then $g_{K}(z, \chi, v)=\sum_{d=0}^{p^{b}-1} \chi(v+d) e^{z d} g_{H_{d}}\left(p^{b} z+i w_{d}\right)$, where $w_{d}:=2 \pi \overline{v+d} L / p^{a-b}$ if $(v+d, p)=1$, with $(\overline{v+d})(v+d) \equiv 1 \bmod p^{a}$, otherwise $w_{d}:=0$. Here, $L \in\left[0, p^{a-b}\right)$ is the integer determined by the equation $\chi\left(1+p^{b}\right)=e^{2 \pi i L / p^{a-b}}$.

The main sum in Theorem 1.2 has $\leq v_{0}+(m+1)(R+1) p^{b}$ terms, where the extra $p^{b}$ is from the formula for $g_{K}(z, \bar{\chi}, v)$ in lemma 1.3. One can easily deduce from the proof of lemma 3.2 that $\mathcal{B}_{M}\left(s, \chi, u_{0}, v_{0}\right) \leq v_{0}^{-\sigma}+\left(M^{1-\sigma}-v_{0}^{1-\sigma}\right)(1-$ $\sigma)^{-1}$ if $\sigma \neq 1$, and $\mathcal{B}_{M}\left(s, \chi, u_{0}, v_{0}\right) \leq v_{0}^{-\sigma}+\log \left(M / v_{0}\right)$ if $\sigma=1$. This bound is generous, of course, and can be improved by computing $\mathcal{B}_{M}\left(s, \chi, u_{0}, v_{0}\right)$ directly, as was pointed out earlier for zeta. In any case, we can ensure that $\left|\mathcal{T}_{M, m}(s, \chi)\right|+$ $\left|\mathcal{R}_{M}(s, \chi)\right|<\epsilon$ for $\sigma=1 / 2$, by taking $M \ll(\mathfrak{q}(s, \chi) / \epsilon)^{2}$ and $m \ll \log (\mathfrak{q}(s, \chi) / \epsilon)$. So, choosing $u_{0}=2\lceil\sqrt{\mathfrak{q}(s)}\rceil$ and $v_{0}=p^{b} u_{0}$, we see that the main sum on the critical line can be made of length $\ll p^{b} \sqrt{\mathfrak{q}(s)} \log ^{2}(\mathfrak{q}(s, \chi) / \epsilon)$ terms. If $a$ is an even integer, or a large integer, then $p^{b} \approx \sqrt{q}$, and so the length of the main sum is about $\sqrt{\mathfrak{q}(s, \chi)} \log ^{2}(\mathfrak{q}(s, \chi) / \epsilon)$. We remark that one can apply the EulerMaclaurin formula along arithmetic progressions to the main sum in (6) (for each 
residue class of $\left.p^{a}\right)$. This way, one can restrict $M \ll \mathfrak{q}(s, \chi)$, replacing $\mathcal{R}_{M}(s, \chi)$ by the correction terms resulting from the Euler-Maclaurin formula. These correction terms will involve sums over the residue classes of $p^{a}$. But it will not be too hard to see that these sums can be tackled using the same methods presented here.

Remark. If $\sigma>0$, then one has the exact expression

$$
L(s, \chi)=\sum_{n=1}^{v_{0}-1} \frac{\chi(n)}{n^{s}}+\sum_{r=0}^{\infty} \frac{1}{v_{r}^{s}} \sum_{j=0}^{\infty} \frac{f_{s}^{(j)}(0)}{j !} \frac{g_{K_{r}}^{(j)}\left(-s / v_{r}, \chi, v_{r}\right)}{v_{r}^{j}} .
$$

The order of the double sum can be switched if $\sigma>1$.

\section{Previous methods And motivation}

In the case of the Riemann zeta function, one can use the Euler-Maclaurin summation to obtain a main sum of length about $\mathfrak{q}(s)$. One notes that $n^{-s}$ changes slowly with $n$ when $n \gg \mathfrak{q}(s)$, and so $n^{-s}$ becomes approximable by the integral $\int_{n}^{n+1} x^{-s} d x$. This gives an efficient way to compute the tail $\sum_{n \gg \mathfrak{q}(s)} n^{-s}$. Specifically, following [13, 14], we have, for any positive integers $N$ and $L_{1}$,

$$
\zeta(s)=\sum_{n=1}^{N-1} n^{-s}+\frac{N^{-s}}{2}+\frac{N^{1-s}}{s-1}+\sum_{\ell=1}^{L_{1}} T_{\ell, N}(s)+E_{N, L_{1}}(s),
$$

where $T_{\ell, N}(s)=\frac{B_{2 \ell}}{(2 \ell) !} N^{-s} \prod_{l=0}^{2 \ell-2}(s+l) / N, B_{2}=1 / 6, B_{4}=-1 / 30, \ldots$, are the Bernoulli numbers, and, by the estimate in [14, we have, for any $\sigma>-\left(2 L_{1}+1\right)$,

$$
\left|E_{N, L_{1}}(s)\right| \leq \frac{\zeta\left(2 L_{1}\right)}{\pi N^{\sigma}} \frac{\left|s+2 L_{1}-1\right|}{\sigma+2 L_{1}-2} \prod_{l=0}^{2 L_{1}-2} \frac{|s+l|}{2 \pi N} .
$$

It follows from (11) that, for $\sigma \geq 1 / 2$ say, one can ensure that $\left|E_{N, L_{1}}(s)\right|<\epsilon$ by taking $2 \pi N \geq e\left|s+2 L_{1}-1\right|$ and $2 L_{1}-1>0.5 \log \left|s+2 L_{1}-1\right|-\log \epsilon$. Therefore, the remainder term in the Euler-Maclaurin summation is easy to control, enabling very accurate computations of zeta.

Rubinstein showed [14 that one could reduce the length of the main sum in the Euler-Maclaurin formula to $\ll \mathfrak{q}(s)^{1 / 2}$ terms, but requiring $\approx \log (\mathfrak{q}(s) / \epsilon) \log (\mathfrak{q}(s))$ precision due to substantial cancellation that occurs, and with each term involving an incomplete Gamma function. The Riemann-Siegel formula offers good control over the required precision, and is often used in zeta computations. The derivation of the Riemann-Siegel formula is quite involved. One begins by expressing $\zeta(s)$ as a contour integral, then moves the contour of integration suitably. This leads to a remainder term that requires careful saddle-point analysis; see [17, Chap. IV] and [6. Chapter 7] for example. One version of the Riemann-Siegel formula on the critical line is the following. For $t>2 \pi$, let $a:=\sqrt{t /(2 \pi)}, n_{1}:=\lfloor a\rfloor$ the integer part of $a$, and $z:=1-2(a-\lfloor a\rfloor)$. Then

$$
e^{i \theta(t)} \zeta(1 / 2+i t)=2 \Re\left(e^{-i \theta(t)} \sum_{n=1}^{n_{1}} \frac{e^{i t \log n}}{\sqrt{n}}\right)-\frac{(-1)^{n_{1}}}{\sqrt{a}} \sum_{r=0}^{m} \frac{C_{r}(z)}{a^{r}}+R_{m}(t) .
$$

The $C_{r}(z)$ can be written as a linear combination of derivatives of the function $F(z):=\cos \left((\pi / 2)\left(z^{2}+3 / 4\right)\right)(\cos (\pi z))^{-1}$ (up to the $3 r$-th derivative). For example, $C_{0}(z)=F(z)$ and $C_{1}(z)=F^{(3)}(z) /\left(12 \pi^{2}\right)$, where $F^{(3)}(z)$ is the third derivative of $F(z)$ with respect to $z$. (Note that $F(z)$ is not periodic in $z$.) The general 
form of $C_{r}(z)$ can be found in Gabcke's thesis [7]. Using formal manipulations of Dirichlet series, Berry showed 2] (see also [3]) that the series of the correction terms $\sum_{r \geq 0} C_{r}(z) a^{-r}$ is divergent, and, therefore, improvement from adding more correction terms in (12) is not to continue indefinitely, instead, the series should be stopped at the least term for a given $t$. The phase $\theta(t)$ is defined by $\theta(t):=$ $\arg \left[\pi^{-i t / 2} \Gamma(1 / 4+i t / 2)\right]$. We can also define $\theta(t)$ by a continuous variation of $s$ in $\pi^{-s / 2} \Gamma(s / 2)$, starting at $s=1 / 2$ and going up vertically, which gives the formula $\theta(t)=(t / 2) \log (t /(2 \pi e))-\pi / 8+1 /(48 t)+O\left(t^{-3}\right)$ for large $t$. We note that the rotation factor $e^{i \theta(t)}$ is chosen so that $e^{i \theta(t)} \zeta(1 / 2+i t)$ is real. Thus, one may locate non-trivial zeros of zeta by looking for sign changes in the r.h.s. of (12).

As for the remainder term $R_{m}(t)$, we have $R_{m}(t) \ll t^{-(2 m+3) / 4}$. Gabcke derived explicit bounds for $R_{m}(t)$, for $m=0, \ldots, 10$ in his thesis [7. For example, for $t \geq$ 200 , we have $\left|R_{1}(t)\right|<.053 t^{-5 / 4},\left|R_{4}(t)\right|<0.017 t^{-11 / 4}$, and $\left|R_{10}(t)\right|<25966 t^{-23 / 4}$. While Gabcke's estimates are sufficient for most applications, they do not allow for very high accuracy for relatively small $t$, such as required when computing zeta zeros to many digits in order to test their linear independence. (Recently, very good bounds have been derived in [1].) A source of the difficulty towards explicit estimates of $R_{m}(t)$ is that the main sum of the Riemann-Siegel formula has a sharp cut-off (dictated by the location of the saddle-point), which complicates the analysis of the remainder term significantly. The analysis is much simplified by using a smoothing function. Indeed, Turing had proposed [18 a type of smoothed formula for computing zeta in the intermediate range where $t$ is neither so small that the Euler-Maclaurin summation can be used nor large enough for the Riemann-Siegel asymptotic formula 2 Rubinstein provides 14 the following smoothed formula, which has a main sum of length $\mathfrak{q}(s)^{1 / 2+o_{\epsilon}(1)}$, and which can be generalized to a fairly large class of $L$-functions.

$\pi^{-s / 2} \Gamma(s / 2) \zeta(s) \delta^{-s}=-\frac{1}{s}-\frac{\delta^{-1}}{1-s}+\sum_{n=1}^{\infty} G\left(s / 2, \pi n^{2} \delta^{2}\right)+\delta^{-1} \sum_{n=1}^{\infty} G\left((1-s) / 2, \pi n^{2} / \delta^{2}\right)$,

where $G(z, w)$ is a smoothing function that can be expressed in terms of the incomplete Gamma function $\Gamma(z, w), G(z, w):=w^{-z} \Gamma(z, w)=\int_{1}^{\infty} e^{-w x} x^{z-1} d x$, $\Re(w)>0$, and $\delta$ is a complex parameter of modulus one, with a simple dependence on $t$, such that $|\Im(\log \delta)| \in(-\pi, \pi]$ and $\Im(\log \delta)$ tends to $\operatorname{sgn}(t) \pi / 4$ for large t. In explicit form, $\delta=\exp (i \operatorname{sgn}(t)(\pi / 4-\theta))$, where $\theta=\pi / 4$ if $|t| \leq 2 c / \pi$, $\theta=c /|2 t|$ if $|t|>2 c / \pi$, and $c>0$ is a free parameter that we can optimize. In particular, $\delta^{-s}$ is chosen to cancel out the exponential decay in $\Gamma(s / 2)$ as $t$ gets large on the l.h.s of (13), ensuring that the l.h.s. is $\gg|s|^{(\sigma-1) / 2}|\zeta(s)| e^{-c}$ for large $t$. Although the series in (13) are infinite, the weights $G(z, w)$ decay exponentially fast when $\Re(w) \gg 1$. Specifically, following [14, we have for $\Re(w)>0$ and $\Re(z) \leq 1$ that $|G(z, w)|<e^{-\Re(w)} / \Re(w)$. So, for $|t|>2 c / \pi$ and $\sigma \in[0,1]$ say, we have $\Re\left(\pi n^{2} \delta^{2}\right)=\Re\left(\pi n^{2} / \delta^{2}\right)=\pi n^{2} \cos (\pi / 2-c /|t|)>\pi n^{2} c /|2 t|$, where we used the inequality $\cos (\pi / 2-x) \geq x / 2$ for $0 \leq x \leq 1$. Therefore, the series can be truncated after $M$ terms with truncation error $<4|t| /(\pi c) \sum_{n>M} n^{-2} e^{-\pi n^{2} c /|2 t|}$. So to ensure that the truncation error is $\langle\epsilon$, it certainly suffices to take $M>$ $\sqrt{|2 t| /(\pi c) \log (|4 t| /(\epsilon c))}$. Once the series is truncated, it can be evaluated term

\footnotetext{
${ }^{2}$ It is worth mentioning that Theorem 1.1 is useful in such a range, in order to carry out high precision computations.
} 
by term to give a numerical approximation of $\zeta(\sigma+i t)$ for $|t|>2 c / \pi$. The number of terms in the resulting main sum (i.e. truncated series) is roughly equal to $\sqrt{\mathfrak{q}(s) \log (\mathfrak{q}(s) / \epsilon)}$. The terms in the main sum are more complicated than in the Riemann-Siegel formula since each term involves the smoothing function $G(z, w)$.

In the case of Dirichlet $L$-functions, Davies [4, Deuring [5], Lavrik [12, and others had developed Riemann-Siegel type formulas for $L(1 / 2+i t, \chi)$, where $\chi$ is a primitive character $\bmod q$ and $t \gg 1$. Such formulas, whose general form was already considered by Siegel [16, require the numerical evaluation of a main sum of length $q\lfloor\sqrt{t /(2 \pi q)}\rfloor \approx \mathfrak{q}(\chi, s)^{1 / 2}$ terms, where each term is of the form $\chi(n) n^{-1 / 2} \exp (i t \log n)$. Unfortunately, however, it does not seem that we have an analogue of Gabcke's explicit estimate for the remainder terms in such formulas. And it is not clear how to obtain a posteriori error estimate either. Therefore, we are not prepared to find the accuracy of the numerics resulting from these formulas explicitly. Still, if one is willing to live with a much longer main sum, consisting of about $\mathfrak{q}(\chi, s)$ terms, then one can keep the simplicity of an unsmoothed main sum while having an explicit estimate for the remainder term. The basic idea is well-known, and was implemented carefully by Rumely [15]. Essentially, one uses the periodicity of $\chi$ to write $L(1 / 2+i t, \chi)$ as a linear combination of about $\mathfrak{q}(\chi, 0)$ Hurwitz zeta functions, then one approximates each Hurwitz zeta function using the Euler-Maclaurin summation formula. However, since the Euler-Maclaurin formula requires a main sum of length about $\mathfrak{q}(s)$, the cost of this method is prohibitive in comparison with a Riemann-Siegel approach with explicit remainder. In view of this, one typically uses a smoothing function to accelerate the convergence. Such formulae (see [14]) are applicable even for small $t$ and have a main sum of length $\mathfrak{q}(\chi, s)^{1 / 2+o_{\epsilon}(1)}$ terms, where each term involves the computation of a smoothing function.

\section{Proofs of Theorems $1.1 \& 1.2$}

We first prove Theorem 1.1. The proof of Theorem 1.2 will be similar, but will additionally require a specialization of the Postnikov character formula, lemma 3.4. Recall that we choose integers $u_{0} \geq 1, v_{0} \geq u_{0}, M \geq v_{0}$, and we construct the sequences $K_{r}=\min \left\{\left\lceil v_{r} / u_{0}\right\rceil, M-v_{r}\right\}$ and $v_{r+1}=v_{r}+K_{r}$ for $0 \leq r \leq R$, where $R:=R\left(v_{0}, u_{0}, M\right)$ is the smallest integer such that $v_{R+1}=M$.

Lemma 3.1. $R=R\left(v_{0}, u_{0}, M\right)<2 u_{0} \log \left(M / v_{0}\right)+1$.

Proof. For $r<R$, we have $v_{r+1}=v_{r}+K_{r} \geq v_{r}\left(1+1 / u_{0}\right)$, and so by induction $v_{r+1} \geq v_{0}\left(1+1 / u_{0}\right)^{r}$. If $R>0$, then taking $r=R-1$ and noting that $v_{R}<M$, we obtain $R<\log \left(M / v_{0}\right) / \log \left(1+1 / u_{0}\right)+1 \leq 2 u_{0} \log \left(M / v_{0}\right)+1$, where we used the inequality $\log (1+x) \geq x / 2$ for $0 \leq x<1$. If $R=0$, then clearly the last bound still holds.

Lemma 3.2. Let $s=\sigma+i t, \sigma \geq 0$. Using the same notation for $K_{r}, v_{r}$, and $R$, we have

$$
\sum_{0 \leq r \leq R} g_{K_{r}}\left(-\sigma / v_{r}\right) v_{r}^{-\sigma} \leq v_{0}^{-\sigma}+ \begin{cases}\frac{M^{1-\sigma}-v_{0}^{1-\sigma}}{1-\sigma}, & \sigma \neq 1, \\ \log \left(M / v_{0}\right), & \sigma=1 .\end{cases}
$$

Proof. For $k<v$, we have $\log (1+k / v)=k / v-k^{2} /\left(2 v^{2}\right)+\cdots \leq k / v$. Thus, $e^{-\sigma k / v} \leq$ $(1+k / v)^{-\sigma}$. Hence, $g_{K}(-\sigma / v) v^{-\sigma} \leq v^{-\sigma} \sum_{0 \leq k<K}(1+k / v)^{-\sigma}=\sum_{0 \leq k<K}(v+k)^{-\sigma}$. 
So $\sum_{0 \leq r \leq R} g_{K_{r}}\left(-\sigma / v_{r}\right) v_{r}^{-\sigma} \leq \sum_{v_{0} \leq n<M} n^{-\sigma} \leq v_{0}^{-\sigma}+\int_{v_{0}}^{M} x^{-\sigma} d x$. The lemma follows on evaluating the integral.

Lemma 3.3. Let $s=\sigma+i t, \sigma \geq 0$. For any integers $v \geq u \geq 2 \max \{6, \sqrt{|s|}, \sigma\}$, $K \geq 1$, and $m \geq 0$, such that $(K-1) / v \leq 1 / u$, we have

$$
\sum_{0 \leq k<K} e^{-s \log (1+k / v)}=\sum_{j=0}^{m} c_{j}(s) \frac{g_{K}^{(j)}(-s / v)}{v^{j}}+\mathcal{E}_{m}(s, v, K),
$$

$c_{j}(s)=\frac{f_{s}^{(j)}(0)}{j !},\left|\mathcal{E}_{m}(s, v, K)\right| \leq \epsilon_{m}(s, u) \min \left\{g_{K}(-\sigma / v),|\csc (t /(2 v))| e^{-\sigma(K-1) / v}\right\}$, and $\epsilon_{m}(s, u)$ is defined in (5).

Proof. We have $e^{-s \log (1+k / v)}=e^{-s k / v} f_{s}(k / v)$. The function $f_{s}(z)$ is analytic in $|z|<1$. Taking the branch of the logarithm determined by $f_{s}(0)=1$, we have $f_{s}(z)=e^{-s \log (1+z)+s z}=e^{s z^{2} / 2-s z^{3} / 3+\cdots}$ for $|z|<1$. We expand $f_{s}(z)$ into a power series $1+\cdots+c_{m}(s) z^{m}+\cdots$. By definition, we have $\mathcal{E}_{m}(s, v, K)=$ $\sum_{0 \leq k<K} e^{-s k / v} \sum_{j>m} c_{j}(s)(k / v)^{j}$. So, interchanging the order of summation in $j$ and $k$, we obtain $\left|\mathcal{E}_{m}(s, v, K)\right| \leq \sum_{j>m}\left|c_{j}(s)\right|\left|\sum_{0 \leq k<K}(k / v)^{j} e^{-s k / v}\right|$. We note that the function $x^{j} e^{-\sigma x}$ is increasing with $x$ if $0 \leq x<j / \sigma$. So, if $0 \leq k<j v / \sigma$, then $(k / v)^{j} e^{-\sigma k / v}$ increases with $k$. This last condition, $k<j v / \sigma$, is satisfied because, by hypothesis, $j>m \geq 0$, so $j \geq 1$, and $k / v \leq 1 / u<1 / \sigma$. Thus, it follows by partial summation that

$$
\left|\mathcal{E}_{m}(s, v, K)\right| \leq e^{-\sigma(K-1) / v} \max _{x \in[0, K]}\left|\sum_{x \leq k<K} e^{-i t k / v}\right| \sum_{j>m}\left|c_{j}(s)\right|(K-1)^{j} / v^{j} .
$$

Executing the summation in the geometric sum, we see that it is bounded by $|\csc (t /(2 v))|$. Also, by a trivial estimate, $\left|\sum_{0 \leq k<K} e^{-s k / v}\right| \leq g_{K}(-\sigma / v)$. Thus,

$$
\left|\mathcal{E}_{m}(s, v, K)\right| \leq \min \left\{g_{K}(-\sigma / v),|\csc (t /(2 v))| e^{-\sigma(K-1) / v}\right\} \sum_{j>m}\left|c_{j}(s)\right| \frac{(K-1)^{j}}{v^{j}} .
$$

We bound $c_{j}(s)$ by a standard application of Cauchy's theorem using a circle around the origin. We have $2 \pi\left|c_{j}(s)\right| \leq\left|\int_{|z|=c} f_{s}(z) / z^{j+1} d z\right| \leq 2 \pi c^{-j} e^{|s| c^{2} / 2+\cdots}, c \in(0,1)$. If $0<j \leq|s| / 4$, let $c=\sqrt{j /|s|} \leq 1 / 2$. So $|s| c^{2} / 2+\cdots \leq|s| c^{2} \sum_{r=2}^{\infty} c^{r-2} / r \leq \alpha j$, where $\alpha:=\sum_{r=2}^{\infty}(1 / 2)^{r-2} / r=-2+4 \log 2<0.78$. We conclude that $\left|c_{j}(s)\right| \leq$ $|s|^{j / 2} j^{-j / 2} e^{\alpha j}$ for $0<j \leq|s| / 4$. Also, for any $j \geq 0$, we may choose $c=1 / 2$. So we have $\left|c_{j}(s)\right| \leq 2^{j} e^{\alpha|s| / 4}$ for each $j \geq 0$.

Since $(K-1) / v \leq 1 / u$, by hypothesis, we have by the estimate for $c_{j}(s)$, and assuming that $m \leq|s| / 4$, that

$$
\sum_{j>m}\left|c_{j}(s)\right| \frac{(K-1)^{j}}{v^{j}} \leq \sum_{m<j \leq|s| / 4}|s|^{j / 2} u^{-j} j^{-j / 2} e^{\alpha j}+\sum_{j>|s| / 4} u^{-j} 2^{j} e^{\alpha|s| / 4} .
$$

If $|s| / 4$ is not an integer, then $\sum_{j>|s| / 4} u^{-j} 2^{j} e^{\alpha|s| / 4} \leq 0.2 e^{\alpha}(u / 2)^{-\lfloor|s| / 4\rfloor} e^{\alpha\lfloor|s| / 4\rfloor}$, where we used $\sum_{\ell>0}(2 / u)^{\ell} \leq 0.2$ and $u \geq 12$. Since this is at most $0.2 e^{\alpha}<0.44$ times the last term in first sum on the r.h.s. above, we obtain the estimate

$$
\sum_{j>m}\left|c_{j}(s)\right| \frac{(K-1)^{j}}{v^{j}} \leq 1.44 \sum_{j>m}|s|^{j / 2} u^{-j} j^{-j / 2} e^{\alpha j}
$$


Now, for $\ell \geq 0,(m+1+\ell)^{-(m+1+\ell) / 2} \leq(m+1)^{-(m+1) / 2}(1+\ell)^{-\ell / 2}$. Therefore,

$$
\sum_{j>m}\left|c_{j}(s)\right| \frac{(K-1)^{j}}{v^{j}} \leq \frac{3.5 e^{\alpha(m+1)}}{(m+1)^{(m+1) / 2}} \frac{|s|^{(m+1) / 2}}{u^{m+1}}<\epsilon_{m}(s, u),
$$

where we used $u \geq 2 \sqrt{|s|}$ and $\sum_{\ell=0}^{\infty} \frac{|s|^{\ell / 2} u^{-\ell} e^{\alpha \ell}}{(1+\ell)^{\ell / 2}} \leq 2.42$, so $(2.42)(1.44)<3.5$. If $|s| / 4$ is an integer, on the other hand, then the same bound holds (with an even better constant). It remains to consider the case when $m>|s| / 4$. Here, we have $\sum_{j>m}\left|c_{j}(s)\right|(K-1)^{j} / v^{j} \leq \sum_{j>m} u^{-j} 2^{j} e^{\alpha|s| / 4} \leq 2^{m} e^{\alpha|s| / 4} / u^{m}$. Therefore, $\sum_{j>m}\left|c_{j}(s)\right|(K-1)^{j} / v^{j} \leq \epsilon_{m}(s, u)$. Put together, we arrive at the claimed bound on $\mathcal{E}_{m}(s, v, K)$. To complete the proof of the lemma, notice that

$$
\sum_{0 \leq k<K} e^{-s \log (1+k / v)}=\sum_{0 \leq k<K} \sum_{j=0}^{m} c_{j}(s)(k / v)^{j} e^{-s k / v}+\mathcal{E}_{m}(s, v, K) .
$$

So the formula (14) follows on interchanging the order of the double sum.

Lemma 3.4. Let $\chi \bmod p^{a}$ be a Dirichlet character, where $p$ is a prime, and let $b=\lceil a / 2\rceil$. Then there exists an integer $L \bmod p^{a-b}$, depending on $\chi, p, a$, and $b$ only (so independent of $x$ ), such that $\chi\left(1+p^{b} x\right)=e^{2 \pi i L x / p^{a-b}}$ for all $x \in \mathbb{Z}$.

Proof. The proof is similar to that of [8, Lemma 4.2], but we still give it here for completeness. Let $H$ be the subgroup in $\left(\mathbb{Z} / p^{a} \mathbb{Z}\right)^{*}$ consisting of the residue classes congruent to $1 \bmod p^{b}$, so $H$ has size $|H|=p^{a-b}$. We identify the elements of $H$ with the set of integers $\left\{1+p^{b} x \mid 0 \leq x<p^{a-b}\right\}$. Consider the function $\psi: H \rightarrow \mathbb{C}$, defined by $\psi\left(1+p^{b} x\right):=e^{2 \pi i x / p^{a-b}}$ By our choice of $b=\lceil a / 2\rceil$, we have $p^{b} \equiv 0 \bmod p^{a-b}$. Therefore, $\psi\left(\left(1+p^{b} x\right)\left(1+p^{b} y\right)\right)=\psi\left(1+p^{b} x\right) \psi\left(1+p^{b} y\right)$ for all $x, y, \in \mathbb{Z}$, meaning that $\psi$ is multiplicative. Also, $\psi$ is not identically zero; e.g. $\psi(1)=1$. Therefore, $\psi$ is a character of $H$. Moreover, the values $\psi\left(1+p^{b}\right)^{u}=$ $e^{2 \pi i u / p^{a-b}}, 0 \leq u<p^{a-b}$, are all distinct. In particular, $\psi$ has order $p^{a-b}$, which is the same as the order of $H$. So $\psi$ generates the full character group of $H$. Since $\left.\chi\right|_{H}$ is a character of $H$, then $\left.\chi\right|_{H} \equiv \psi^{L}$ for some $L \bmod p^{a-b}$. To find $L$, we calculate $\chi\left(1+p^{b}\right)$, then use the relation $\chi\left(1+p^{b}\right)=e^{2 \pi i L / p^{a-b}}$.

Proof of Theorem 1.1. We divide the main sum in (11) according to the positions of $v_{r}$ as follows: $\sum_{1 \leq n<v_{0}} n^{-s}+\sum_{0 \leq r \leq R} v_{r}^{-s} \sum_{0 \leq k<K_{r}} e^{-s \log \left(1+k / v_{r}\right)}$. Note that $K_{r}=\left\lceil v_{r} / u_{0}\right\rceil \leq v_{r} / u_{0}+1$ for $r<R$, and $K_{R} \leq v_{R} / u_{0}+1$. So $\left(K_{r}-1\right) / v_{r} \leq 1 / u_{0}$ throughout $0 \leq r \leq R$. Thus, the conditions for lemma 3.3 are satisfied and we can apply it to each block $\sum_{0 \leq k<K_{r}} e^{-s \log \left(1+k / v_{r}\right)}$. This yields $\mathcal{T}_{M, m}\left(s, u_{0}, v_{0}\right)=$ $\sum_{0 \leq r \leq R} v_{r}^{-s} \mathcal{E}_{m}\left(s, v_{r}, K_{r}\right)$. And using the estimate for $\mathcal{E}_{m}(s, v, K)$ in lemma 3.3 yields the required bound on $\mathcal{T}_{M, m}\left(s, u_{0}, v_{0}\right)$. 
Proof of Lemma 1.3. This follows from from the definitions and lemma 3.4:

$$
\begin{aligned}
g_{K}(z, \chi, v) & =\sum_{d=0}^{p^{b}-1} \sum_{0 \leq k<H_{d}} e^{z\left(d+p^{b} k\right)} \chi\left(v+d+p^{b} k\right) \\
& =\sum_{d=0}^{p^{b}-1} \delta_{\operatorname{gcd}(v+d, p)=1} \chi(v+d) e^{z d} \sum_{0 \leq k<H_{d}} e^{p^{b} z k} \chi\left(1+p^{b} \overline{v+d} k\right) \\
& =\sum_{d=0}^{p^{b}-1} \delta_{\operatorname{gcd}(v+d, p)=1} \chi(v+d) e^{z d} \sum_{0 \leq k<H_{d}} e^{p^{b} z k+2 \pi i L \overline{v+d} k / p^{a-b}} \\
& =\sum_{d=0}^{p^{b}-1} \chi(v+d) e^{z d} g_{H_{d}}\left(p^{b} z+i w_{d}\right) .
\end{aligned}
$$

Lemma 3.5. Given $s=\sigma+i t, \sigma \geq 0$, and a Dirichlet character $\chi \bmod p^{a}$ with $p$ a prime, let $b=\lceil a / 2\rceil$. Then for any integers $v \geq u \geq 2 \max \{6, \sqrt{|s|}, \sigma\}, K \geq 1$, and $m \geq 0$, such that $(K-1) / v \leq 1 / u$, we have

$$
\sum_{0 \leq k<K} \chi(v+k) e^{-s \log (1+k / v)}=\sum_{j=0}^{m} c_{j}(s) \frac{g_{K}^{(j)}(-s / v, \chi, v)}{v^{j}}+\mathcal{E}_{m}(s, \chi, v, K),
$$

where $c_{j}(s)=\frac{f_{s}^{(j)}(0)}{j !}$, and, with $H_{d}=\left\lceil(K-d) / p^{b}\right\rceil$, we have

$$
\left|\mathcal{E}_{m}(s, \chi, v, K)\right| \leq \epsilon_{m}(s, u) \sum_{d=0}^{p^{b}-1} \delta_{\operatorname{gcd}(v+d, p)=1} \min \left\{g_{H_{d}}\left(-p^{b} \sigma / v\right),\left|\csc \left(w_{d} / 2-p^{b} t /(2 v)\right)\right|\right\} .
$$

The $\epsilon_{m}(s, u)$ is defined in (5).

Proof. Proceeding in the same way as in Theorem 1.1 and lemma 1.3, we arrive at

$$
\begin{aligned}
& \mathcal{E}_{m}(s, \chi, v, K)=\sum_{0 \leq k<K} \chi(v+k) e^{-s k / v} \sum_{j>m} c_{j}(s)(k / v)^{j} \\
& =\sum_{j>m} c_{j}(s) \sum_{0 \leq k<K} \chi(v+k) e^{-s k / v}(k / v)^{j} \\
& =\sum_{j>m} c_{j}(s) \sum_{d=0}^{p^{b}-1} \sum_{0 \leq k<H_{d}} \chi\left(v+d+p^{b} k\right) e^{-s\left(d+p^{b} k\right) / v}\left(\left(d+p^{b} k\right) / v\right)^{j} \\
& =\sum_{j>m} c_{j}(s) \sum_{d=0}^{p^{b}-1} \chi(v+d) \sum_{\ell=0}^{j}\left(\begin{array}{l}
j \\
\ell
\end{array}\right) d^{j-\ell} v^{\ell-j} e^{-s d / v} \sum_{0 \leq k<H_{d}}\left(p^{b} k / v\right)^{\ell} e^{\left(-p^{b} s / v+i w_{d}\right) k} .
\end{aligned}
$$


Therefore, using partial summation, as in the proof of lemma 3.3, we obtain

$$
\begin{aligned}
& \left|\mathcal{E}_{m}(s, \chi, v, K)\right| \\
& \leq \sum_{j>m}\left|c_{j}(s)\right| \sum_{d=0}^{p^{b}-1} \delta_{\operatorname{gcd}(v+d, p)=1} \sum_{\ell=0}^{j}\left(\begin{array}{l}
j \\
\ell
\end{array}\right)(d / v)^{j-\ell} e^{-\sigma d / v}\left|\sum_{0 \leq k<H_{d}}\left(p^{b} k / v\right)^{\ell} e^{\left(-p^{b} s / v+i w_{d}\right) k}\right| \\
& \leq \sum_{j>m}\left|c_{j}(s)\right| \sum_{d=0}^{p^{b}-1} \delta_{\operatorname{gcd}(v+d, p)=1}\left(\left(d+p^{b}\left(H_{d}-1\right)\right) / v\right)^{j} \max _{x \in\left[0, H_{d}\right]}\left|\sum_{x \leq k<H_{d}} e^{i\left(-p^{b} t / v+w_{d}\right) k}\right| \\
& \leq \sum_{j>m}\left|c_{j}(s)\right|(K-1)^{j} / v^{j} \sum_{d=0}^{p^{b}-1} \delta_{\operatorname{gcd}\left(v_{r}+d, p\right)=1} \max _{x \in\left[0, H_{d}\right]}\left|\sum_{x \leq k<H_{d}} e^{i\left(-p^{b} t / v+w_{d}\right) k}\right| \\
& \leq \epsilon_{m}(s, u) \sum_{d=0}^{p^{b}-1} \delta_{\operatorname{gcd}\left(v_{r}+d, p\right)=1}\left|\csc \left(w_{d} / 2-p^{b} t /(2 v)\right)\right| .
\end{aligned}
$$

Combined with the trivial estimate, this yields the lemma.

Proof of Theorem 1.2. We divide the main sum in (6) according to the positions of $v_{r}$ as before: $\sum_{1<n<v_{0}} \chi(n) n^{-s}+\sum_{0 \leq r \leq R} v_{r}^{-s} \sum_{0 \leq k<K_{r}} \chi(v+r) e^{-s \log \left(1+k / v_{r}\right)}$. We apply lemmas 3.5 and 1.3 to the sum over $k$. This yields the result with $\mathcal{T}_{M, m}\left(s, \chi, u_{0}, v_{0}\right)=\sum_{0 \leq r \leq R} v_{r}^{-s} \mathcal{E}_{m}\left(s, \chi, v_{r}, K_{r}\right)$. By the estimate for $\mathcal{E}_{m}(s, \chi, v, K)$ in lemma 3.5 we obtain the desired bound on $\mathcal{T}_{M, m}\left(s, \chi, u_{0}, v_{0}\right)$.

$$
\text { 4. COMPuting } \frac{f_{s}^{(j)}(0)}{j !} \frac{g_{K}^{(j)}(z)}{v^{j}} \text { FOR } 0 \leq j \leq m
$$

One can choose the parameters in Theorems $1.1 \& 1.2$ so that one can achieve moderate accuracy with $m \leq 8$, say. So, in general, computing $\left(f_{s}^{(j)}(0) / j !\right)\left(g_{K}^{(j)}(z) / v^{j}\right)$ will be quite easy, and can be done using closed-form formulas to evaluate the geometric sum. The methods that we present below are intended for when $j$ is large, but they can be used for any $j \geq 0$. In our application (Theorems 1.1 \& 1.2), we have $(K-1) / v \leq 1 / u_{0} \leq 1 /(2 \sqrt{\mathfrak{q}(s)})$, and $-1 / 2 \leq \Re(z) \leq 0$. So we will assume that this holds throughout.

We recall that $f_{s}(z)=e^{s z^{2} / 2-s z^{3} / 3+\cdots}=\sum_{j \geq 0} \frac{f_{s}^{(j)}(0)}{j !} z^{j}$ for $|z|<1$. For example,

$$
\begin{aligned}
& f_{s}(0)=1, \quad f_{s}^{(1)}(0)=0, \quad f_{s}^{(2)}(0)=s, \quad f_{s}^{(3)}(0)=-2 s, \\
& f_{s}^{(4)}(0)=3 s(2+s), \quad f_{s}^{(5)}(0)=-4 s(6+5 s), \\
& f_{s}^{(6)}(0)=5 s\left(24+26 s+3 s^{2}\right), \quad f_{s}^{(7)}(0)=-6 s\left(120+154 s+35 s^{2}\right), \\
& f_{s}^{(8)}(0)=7 s\left(720+1044 s+340 s^{2}+15 s^{3}\right), \quad \ldots
\end{aligned}
$$

To find $f_{s}^{(j)}(0)$ in general, let $q(z):=\sum_{\alpha \geq 2}(-1)^{\alpha} z^{\alpha} / \alpha$, so $f_{s}^{(j)}(z)=Q_{s, j}(z) e^{s q(z)}$ for some $Q_{s, j}(z)=\sum_{l \geq 0} w_{j, l}(s) z^{l}$ that satisfies the recursion $Q_{s, 0}(z):=1$ and $Q_{s, j+1}(z)=\frac{d}{d z} Q_{s, j}(z)+s Q_{s, j}(z) \frac{d}{d z} q(z)$. Therefore, $w_{j, 0}(s)=f_{s}^{(j)}(0), w_{0,0}(s)=1$, $w_{0, l}(s)=0$ for $l>0$, and $w_{j+1, l}(s)=(l+1) w_{j, l+1}(s)-s \sum_{\alpha=1}^{l}(-1)^{\alpha} w_{j, l-\alpha}(s)$. Using this recursion, one can find all of $f_{s}^{(j)}(0)=w_{j, 0}(s)$ for $0 \leq j \leq m$ in about $(m+1)^{2}$ steps. In carrying out the recursion, one may treat $s$ symbolically, so 
$w_{j, 0}(s)$ is viewed as a polynomial in $s$ and the recursion is finding the coefficients of this polynomial. In fact, it follows from the recursion that, more generally, $w_{j, l}(s)$ is a polynomial in $s$ of degree $\leq \min \{(j+l) / 2, j\}$. So we may write $w_{j, l}(s)=$ $\sum_{0 \leq \eta \leq j / 2} \beta_{j, l, \eta} s^{\eta}$. Also, $\beta_{0,0,0}=1, \beta_{0,0, \eta}=0$ for $\eta>0, \beta_{0, l, \eta}=0$ for $l>0$, and we have $\beta_{j+1, l, \eta}=(l+1) \beta_{j, l+1, \eta}-\sum_{\alpha=1}^{l}(-1)^{\alpha} \beta_{j, l-\alpha, \eta-1}$. Therefore, using induction, we obtain the bound $\left|\beta_{j, l, \eta}\right| \leq(j+l) ! 2^{l+1} / l$ !. In particular, $\left|\beta_{j, 0, \eta}\right| / j ! \leq 2$. Thus, the number of bits needed to represent $\left|\beta_{j, 0, \eta}\right| / j$ !, and hence to compute $f_{s}^{(j)}(0) / j$ ! as a polynomial in $s$, to a given precision, is also well-controlled.

As for computing $g_{K}^{(j)}(z)$, one can use the formula $g_{K}^{(j)}(z)=\sum_{\ell=0}^{j}\left(\begin{array}{l}j \\ \ell\end{array}\right) w^{(j-\ell)}(z) y^{(\ell)}(z)$, where $w(z):=e^{K z}-1$ and $y(z):=\left(e^{z}-1\right)^{-1}$. So for $z \notin 2 \pi i \mathbb{Z}$ we have

$$
\begin{aligned}
\frac{f_{s}^{(j)}(0)}{j !} \frac{g_{K}^{(j)}(z)}{v^{j}} & =\frac{f_{s}^{(j)}(0)}{j !} \frac{2^{j}(K-1)^{j}}{v^{j}} \frac{g_{K}^{(j)}(z)}{2^{j}(K-1)^{j}} \\
& =\frac{f_{s}^{(j)}(0)}{j !} \frac{2^{j}(K-1)^{j}}{v^{j}}\left(e^{K z} \sum_{\ell=0}^{j} \frac{1}{2^{j}}\left(\begin{array}{l}
j \\
\ell
\end{array}\right) \frac{y^{(\ell)}(z)}{(K-1)^{\ell}}-\frac{y^{(j)}(z)}{(K-1)^{j}}\right) .
\end{aligned}
$$

The factor $2^{-j}$ is inserted inside the sum in (27) in order to to control the size of the binomial coefficient $\left(\begin{array}{l}j \\ l\end{array}\right) \leq 2^{j}$. By hypothesis, $(K-1) / v \leq 1 / u_{0} \leq 1 /(2 \sqrt{\mathfrak{q}(s)})$. So, recalling that $f_{s}^{(j)}(0)=\sum_{0 \leq \eta \leq j / 2} \beta_{j, 0, \eta} s^{\eta},\left|\beta_{j, 0, \eta}\right| \leq 2(j !)$, and $\mathfrak{q}(s) \geq 3$, we obtain $\left|f_{s}^{(j)}(0) 2^{j}(K-1)^{j}\right| /\left(j ! v^{j}\right) \leq 5$. In particular, the number of bits needed to represent the outside factor in (27) is well-controlled, and we may focus on computing the sum enclosed in parentheses.

To that end, we consider the computation of $y^{(\ell)}(z) /(K-1)^{\ell}$ in (27). If $\ell$ is small, this can be done by directly differentiating $y(z)$, but this is not a practical method if $\ell$ is large. Instead, we note that $z y(z)=z /\left(e^{z}-1\right)$ is the exponential generating function for the Bernoulli numbers, specifically,

$$
y(z)=\frac{1}{z}-\frac{1}{2}+\sum_{l=1}^{\infty} \frac{B_{2 l}}{(2 l) !} z^{2 l-1}, \quad 0<|z|<2 \pi .
$$

Therefore, for $\ell>0$,

$$
y^{(\ell)}(z)=\frac{(-1)^{\ell} \ell !}{z^{\ell+1}}+\sum_{l=\lceil(\ell+1) / 2\rceil}^{\infty} \frac{B_{2 l}}{2 l} \frac{z^{2 l-\ell-1}}{(2 l-\ell-1) !}, \quad 0<|z|<2 \pi .
$$

Using the periodicity of $e^{z}$, and our assumption on $z$, we can ensure that the argument given to $g_{K}^{(j)}(z)$ satisfies $|z|<3 \pi / 2$. Thus, the above formulas will suffice to compute $y^{(\ell)}(z) /(K-1)^{\ell}$ provided that $|z|$ is sufficiently bounded away from 0 , say $|z|>(m+1) /(K-1)$. For such $z$, and assuming that $K>2 \pi(m+1)$ (otherwise, we may compute $g_{K}^{(j)}(z)$ by direct summation in $\ll m+1$ steps), we obtain that $y^{(\ell)}(z) /(K-1)^{\ell}$ is bounded by a constant, and so its size is wellcontrolled. Thus, the only remaining case is when $|z|<(m+1) /(K-1)$, with $K>2 \pi(m+1)$. In this case, we use the Euler-Maclaurin summation. To this end, let $h_{j, z}(x):=x^{j} e^{z x}$. Then $g_{K}^{(j)}(z)=\sum_{0 \leq k<K} h_{j, z}(k)$. Note that, using the periodicity of $e^{z k}$ and conjugating if necessary, we may assume that $0 \leq \Im(z) \leq \pi$. 
By the Euler-Maclaurin formula (see [14]), we have

$$
\begin{aligned}
g_{K}^{(j)}(z) & =\int_{0}^{K-1} h_{j, z}(x) d x+\sum_{\ell=1}^{L} \frac{B_{2 \ell}}{(2 \ell) !}\left(h_{j, z}^{(2 \ell-1)}(K-1)-h_{j, z}^{(2 \ell-1)}(0)\right) \\
& +\frac{1}{2}\left(h_{j, z}(K-1)+h_{j, z}(0)\right)+\mathcal{E}_{K, j, z, L},
\end{aligned}
$$

where $h_{j, z}^{(2 \ell-1)}(x)$ is the $(2 \ell-1)$-st derivative of $h_{j, z}(x)$ with respect to $x$, and the remainder term $\mathcal{E}_{K, j, z, L}=(-1 /(2 L) !) \int_{0}^{K-1} B_{2 L}(\{x\}) h_{j, z}^{(2 L)}(x) d x$, where $B_{2 L}(x)$ is the $2 L$-th Bernoulli polynomial (e.g. $B_{2}(x)=x^{2}-x+1 / 6$ ), and $\{x\}$ is the factional part of $x$. Now, $h_{j, z}^{(2 \ell-1)}(x)=\sum_{l=0}^{2 \ell-1}\left(\begin{array}{c}2 \ell-1 \\ l\end{array}\right)\left(\frac{d^{l}}{d x} x^{j}\right)\left(\frac{d^{2 \ell-l-1}}{d x^{2 \ell-l-1}} e^{z x}\right)$. Thus, we have

$$
h_{j, z}^{(2 \ell-1)}(x)=e^{z x} \sum_{l=0}^{\min \{2 \ell-1, j\}}\left(\begin{array}{c}
2 \ell-1 \\
l
\end{array}\right) \frac{j !}{(j-l) !} x^{j-l} z^{2 \ell-l-1} .
$$

Also, from the Fourier expansion for $B_{2 L}(\{x\})$ (see [14), $\left|B_{2 L}(\{x\})\right| \leq 4 \frac{(2 L) !}{(2 \pi)^{2 L}}$. Therefore, since $\Re(z) \leq 0$, we deduce that $\left|\mathcal{E}_{K, j, z, L}\right| /(K-1)^{j} \leq 4(K-1)(2 \pi)^{-2 L}$, which decays exponentially with $L$.

As for the main term $\int_{0}^{K-1} h_{j, z}(x) d x$ in formula (30), its computation does not present any difficulty since $|z|<m / K$ (so $z$ is small). For example, one can split the interval of integration into $m+1$ consecutive subintervals of equal length, then, after a suitable change of variable, apply Taylor expansions to the integrand in each subinterval, which reduces the problem to integrating polynomials. Alternatively, one can use a numerical quadrature rule.

\section{A CONVEXITY BOUND}

We will use the following well-spacing lemma to prove corollary 5.2 .

Lemma 5.1. Let $\left\{x_{n}, n=0,1, \ldots\right\}$ be a set of real numbers. Suppose there exists a positive integer $Q$ such that $\min _{n \neq n^{\prime}}\left|x_{n}-x_{n^{\prime}}\right| \geq 1 /(2 Q)$. Then, for any $y \geq x$ and any $P \geq 1$, we have

$$
\sum_{x_{n} \in[x, y]} \min \left\{P,\left|\csc \left(\pi x_{n}\right)\right|\right\} \leq(1+\lfloor y-x\rfloor)(2(A+1) P+2 Q \log (Q / A)),
$$

where $A$ is any positive integer that satisfies $A \leq Q / P$.

Proof. Since $\left|x_{n}-x_{n^{\prime}}\right| \geq 1 /(2 Q)$ for $n \neq n^{\prime}$, then for any integer $k$ we have $\sum_{x_{n} \in[k-1 / 2, k+1 / 2]} \min \left\{P,\left|\csc \left(\pi x_{n}\right)\right|\right\} \leq P+\sum_{|l| \leq Q} \min \{P,|\csc (\pi l /(2 Q))|\}=: *$. Using the inequality $|\sin (\pi \alpha)| \geq 2|\alpha|,-1 / 2 \leq \alpha \leq 1 / 2$, we obtain that $* \leq 2(A+$ 1) $P+\sum_{A<|l| \leq Q} Q /|l|$. Combined with the inequality $\sum_{A<l \leq Q} 1 / l \leq \log (Q / A)$, this gives $* \leq 2(A+1) P+2 Q \log (Q / A)$. Since the interval $[x, y]$ contains $\leq 1+\lfloor y-x\rfloor$ integers, the lemma follows.

The bound that we obtain in corollary 5.2 for zeta is, of course, superseded by the bound that one can obtain from the Riemann-Siegel formula. Nevertheless, it illustrates that Theorem 1.1 yields a convexity bound of similar strength to the Riemann-Siegel formula, up to a constant factor, even though it is quite elementary.

Corollary 5.2. $|\zeta(1 / 2+i t)| \ll \mathfrak{q}(1 / 2+i t)^{1 / 4}$. 
Proof. We will use Theorem 1.1, but replacing $\mathcal{R}_{M}(s)$ by the correction terms from the Euler-Maclaurin formula for $\zeta(s)$ (see the paragraph following the statement of the theorem). We take $s=1 / 2+i t, m=0, v_{0}=u_{0}=4\lceil\sqrt{t}\rceil, M=10\lceil t\rceil$, and assume that $t \geq 36$, as we may. Given our choice of $M$, it is not hard to show that the Euler-Maclaurin correction terms contribute $\ll 1$. And given our choice of $u_{0}$, we have $\epsilon_{0}\left(s, u_{0}\right) \ll 1$. By routine calculations, $\sum_{n=1}^{v_{0}-1} n^{-1 / 2} \leq 2 \sqrt{v_{0}}$ and $\left|\sum_{r=0}^{R} g_{K_{r}}\left(-s / v_{r}\right) v_{r}^{-s}\right| \leq \mathcal{B}_{M}\left(s, u_{0}, v_{0}\right)$. Thus,

$$
|\zeta(1 / 2+i t)| \ll \sqrt{v_{0}}+\mathcal{B}_{M}\left(1 / 2+i t, u_{0}, v_{0}\right) .
$$

It is helpful to recall that $K_{r}=\left\lceil v_{r} / u_{0}\right\rceil$ for $r<R, v_{r+1}=v_{r}+K_{r}$, and $\mathcal{B}_{M}\left(s, u_{0}, v_{0}\right) \leq \sum_{r=0}^{R} \min \left\{K_{r},\left|\csc \left(t /\left(2 v_{r}\right)\right)\right|\right\} v_{r}^{-\sigma}$. So, letting $I_{\ell}:=\left(2^{\ell} u_{0}, 2^{\ell+1} u_{0}\right]$, we see that if $v_{r} \in I_{\ell}$, then $2^{\ell}<K_{r} \leq 2^{\ell+1}$. We let $I_{\ell_{0}}$ denote the interval containing $M$, so $M \leq 2^{\ell_{0}+1} u_{0}$. Then, using simple estimates, we obtain

$$
\mathcal{B}_{M}\left(s, u_{0}, v_{0}\right) \leq u_{0}^{-1 / 2} \sum_{\ell=0}^{\ell_{0}} 2^{-\ell / 2} \sum_{v_{r} \in I_{\ell}} \min \left\{2^{\ell+1}, \mid \csc \left(t /\left(2 v_{r}\right) \mid\right\} .\right.
$$

Now, consider that for $v_{r}, v_{r+1} \in I_{\ell}$, with $r<R$, we have

$$
\frac{t}{2 v_{r}}-\frac{t}{2 v_{r+1}}=\frac{t K_{r}}{2 v_{r} v_{r+1}} \geq \frac{t 2^{\ell}}{2^{2 \ell+3} u_{0}^{2}} \geq \frac{t}{2^{\ell+3} u_{0}^{2}} \geq \frac{\pi}{B 2^{\ell+2}}
$$

where $B$ is the smallest positive integer such that $t /\left(2 \pi u_{0}^{2}\right) \geq 1 / B$. Note that, since $u_{0}=4\lceil\sqrt{t}\rceil$, then $B \ll 1$. Also, as $v_{r}$ ranges over $I_{\ell}$, the argument $t /\left(2 \pi v_{r}\right)$ moves by increments $\geq 1 /\left(B 2^{\ell+2}\right)$, and it spans an interval of length $\leq t /\left(2 \pi 2^{\ell} u_{0}\right)-$ $t /\left(2 \pi 2^{\ell+1} u_{0}\right)=t /\left(\pi 2^{\ell+2} u_{0}\right)$. Therefore, applying lemma [5.1 to the set $\left\{v_{r} \in I_{\ell}\right\}$ with $Q=B 2^{\ell+1}, A=B$, and $P=2^{\ell+1}$, we obtain

$$
\begin{aligned}
\sum_{v_{r} \in I_{\ell}} \min \left\{2^{\ell+1}, \mid \csc \left(t /\left(2\left(v_{r}\right)\right) \mid\right\} \leq\right. & \left(1+t /\left(\pi 2^{\ell+2} u_{0}\right)\right)\left(2(B+1) 2^{\ell+1}\right. \\
& \left.+B 2^{\ell+2} \log \left(2^{\ell+1}\right)\right) \ll t(\ell+1) / u_{0} .
\end{aligned}
$$

It follows that $\mathcal{B}_{M}\left(s, u_{0}, v_{0}\right) \leq\left(t /\left(u_{0} \sqrt{v_{0}}\right) \sum_{\ell=0}^{\ell_{0}}(\ell+1) / 2^{-\ell / 2} \ll t /\left(u_{0} \sqrt{v_{0}}\right)\right.$. So we conclude, $\zeta(1 / 2+i t) \ll \sqrt{v_{0}}+t /\left(u_{0} \sqrt{v_{0}}\right) \ll t^{1 / 4}$.

\section{Parameter choices}

Theorem 1.1 offers a simple method for computing $\zeta(\sigma+i t)$ with an explicit error bound. The control over the error term in the theorem goes beyond what the Riemann-Siegel asymptotic formula enables. Theorem 1.2 achieves the same for $L(\sigma+i t, \chi)$ when $\chi$ is power-full.

We implemented a basic version of Theorem 1.1 in Mathematica 9, which is an application for computation, see http://www.wolfram.com/mathematica/. This was sufficient for our purposes as we were mainly interested in learning about reasonable choices of the parameters. This way, we could appraise the accuracy and running time in practice. The Mathematica notebook containing the implementation is available at https://people.math.osu.edu/hiary.1/

Our computation relies on finite precision arithmetic, which introduces roundoff errors. Such errors become significant for large $t$. This is primarily because the computation of $t \log n \bmod 2 \pi$ will contain only a few correct digits for large 
$t$. In general, one cannot expect more than $\pm \epsilon_{\text {mach }} t \log n$ accuracy when computing $e^{i t \log n}$, where $\epsilon_{\text {mach }}$ is the machine epsilon. So if $t>1 / \epsilon_{\text {mach }}$ say, then, certainly, numerical results will not be meaningful. To overcome this problem, one could switch to an arithmetic system with a smaller machine epsilon (but having a slower performance). Assuming that round-off errors behave like independent random variables, which is a reasonable model, the accumulated round-off error in computing $\sum_{n<M} n^{-1 / 2-i t}$ will be typically like $\pm \epsilon_{\operatorname{mach}} t\left(\sum_{n<M}(\log n)^{2} / n\right)^{1 / 2}$. For double-precision arithmetic, $\epsilon_{\text {mach }}=2^{-52} \approx 2 \times 10^{-16}$. So, if we use doubleprecision arithmetic with $t=10^{d}$ and $M \approx 10 t$, the accumulated round-off error will be like $\pm 10^{d-16} \log 10^{3(d+1) / 2}$.

With this in mind, we obtained marginally better control over the round-off errors by using the main sum from the Euler-Maclaurin formula with 6 correction terms, and with $M=10\lceil\mathfrak{q}(s)\rceil$, in particular we did not need to take $M$ very large. We computed $g_{K}(z)$ using the formula $\left(e^{K z}-1\right) /\left(e^{z}-1\right)$ when $|z|>10(m+1) /(K-$ 1) (as is typically the case), and using the Mathematica built-in Euler-Maclaurin summation routine when $|z|<10(m+1) /(K-1)$. To check the accuracy of the results, we compared them with the outputs from lcalc and the Mathematica built-in zeta routine, leading to Table 1 . We attempted to increase the accuracy by inputting $t$ in Mathematica using a higher precision. However, it is likely that Mathematica still uses double-precision arithmetic in intermediate steps and some built-in routines. So the accuracy of many stages of the computation will be limited by the machine epsilon for double-precision numbers.

The error entries in Table 1 are significantly smaller than the explicit bound for $\mathcal{T}_{M, m}\left(s, u_{0}, v_{0}\right)$ given in Theorem 1.1. For example, when $t=10^{10}$ and $m=6$, the explicit bound gives $\left|\mathcal{T}_{M, m}\left(s, u_{0}, v_{0}\right)\right| \leq 2.9 \times 10^{-3}$ (here, we calculated $\mathcal{B}_{M}(s, u 0, v 0)$ directly). This is significantly larger than the observed error $1.9 \times 10^{-10}$ in Table 1 This is not surprising, and is due to the pseudo-random nature of round-off errors.

TABle 1. Error for various $t$ and $m$, and using $\sigma=1 / 2, u_{0}=6\lceil\sqrt{\mathfrak{q}(s)}$
$v_{0}=10(m+1) u_{0}$.
\begin{tabular}{lllll}
$t$ & $m=0$ & $m=2$ & $m=4$ & $m=6$ \\
\hline $10^{4}$ & $3.0 \times 10^{-4}$ & $1.7 \times 10^{-6}$ & $5.8 \times 10^{-9}$ & $3.7 \times 10^{-11}$ \\
$10^{6}$ & $1.2 \times 10^{-2}$ & $1.6 \times 10^{-5}$ & $7.0 \times 10^{-9}$ & $6.9 \times 10^{-12}$ \\
$10^{8}$ & $1.9 \times 10^{-2}$ & $2.7 \times 10^{-5}$ & $2.8 \times 10^{-7}$ & $9.4 \times 10^{-10}$ \\
$10^{10}$ & $5.4 \times 10^{-3}$ & $1.6 \times 10^{-5}$ & $4.2 \times 10^{-8}$ & $1.9 \times 10^{-10}$
\end{tabular}

There was no attempt to optimize our implementation since, in any case, it is not competitive with an implementation directly in $\mathrm{C} / \mathrm{C}++$. With our parameter choices, and for large $t$, the implementation was slower by factor of about $2(m+1)^{2} \log t$ compared to computing the main sum in a Riemann-Siegel formula directly (in both cases we input $t$ in higher precision than double-precision). The implementation was faster by a factor of about $10 \sqrt{t} /\left((m+1)^{2} \log t\right)$ than computing $\sum_{n \leq M} n^{-s}$ directly (this is essentially the main sum in the Euler-Maclaurin formula). It might be possible to speed up the implementation by a factor of $m+1$ if the derivatives $g_{K}^{(j)}(z), 0 \leq j \leq m$, are computed simultaneously via a recursion. One can also save a factor of 2 by choosing $u_{0}=3\lceil\sqrt{\mathfrak{q}(s)}\rceil$ instead of $u_{0}=6\lceil\sqrt{\mathfrak{q}(s)}\rceil$, at the expense of a larger truncation error $\mathcal{T}_{M, m}\left(s, u_{0}, v_{0}\right)$. 


\section{REFERENCES}

1. J. Arias de Reyna, High precision computation of Riemann's zeta function by the RiemannSiegel formula, I, Math. Comp. 80 (2011), no. 274, 995-1009. MR 2772105 (2012c:11171)

2. M. V. Berry, The Riemann-Siegel expansion for the zeta function: high orders and remainders, Proc. Roy. Soc. London Ser. A 450 (1995), no. 1939, 439-462. MR 1349513 (96f:11105)

3. M. V. Berry and J. P. Keating, A new asymptotic representation for $\zeta\left(\frac{1}{2}+i t\right)$ and quantum spectral determinants, Proc. Roy. Soc. London Ser. A 437 (1992), no. 1899, 151-173. MR 1177749 (93j:11057)

4. D. Davies, An approximate functional equation for Dirichlet L-functions, Proc. Roy. Soc. Ser. A 284 (1965), 224-236. MR 0173352 (30 \#3565)

5. Max Deuring, Asymptotische Entwicklungen der Dirichletschen L-Reihen, Math. Ann. 168 (1967), 1-30. MR 0213309 (35 \#4173)

6. H. M. Edwards, Riemann's zeta function, Dover Publications Inc., Mineola, NY, 2001, Reprint of the 1974 original [Academic Press, New York; MR0466039 (57 \#5922)]. MR 1854455 (2002g:11129)

7. W. Gabcke, Neue herleitung und explicite restabschätzung der riemann-siegel-formel., Ph.D. thesis, Göttingen, 1979.

8. Ghaith A. Hiary, Computing dirichlet character sums to a power-full modulus, arXiv:1205.4687 [math.NT] (2012), 23, To appear in the Journal of Number Theory.

9. Ghaith Ayesh Hiary, Fast methods to compute the Riemann zeta function, Ann. of Math. (2) 174 (2011), no. 2, 891-946. MR 2831110 (2012g:11154)

10. H. Iwaniec and P. Sarnak, Perspectives on the analytic theory of L-functions, Geom. Funct. Anal. (2000), no. Special Volume, Part II, 705-741, GAFA 2000 (Tel Aviv, 1999). MR 1826269 (2002b:11117)

11. Henryk Iwaniec and Emmanuel Kowalski, Analytic number theory, American Mathematical Society Colloquium Publications, vol. 53, American Mathematical Society, Providence, RI, 2004. MR 2061214 (2005h:11005)

12. A. F. Lavrik, The approximate functional equation for Dirichlet L-functions, Trudy Moskov. Mat. Obšč 18 (1968), 91-104. MR 0236126 (38 \#4424)

13. A. M. Odlyzko and A. Schönhage, Fast algorithms for multiple evaluations of the Riemann zeta function, Trans. Amer. Math. Soc. 309 (1988), no. 2, 797-809. MR 961614 (89j:11083)

14. Michael Rubinstein, Computational methods and experiments in analytic number theory, Recent perspectives in random matrix theory and number theory, London Math. Soc. Lecture Note Ser., vol. 322, Cambridge Univ. Press, Cambridge, 2005, pp. 425-506. MR 2166470 (2006d:11153)

15. Robert Rumely, Numerical computations concerning the ERH, Math. Comp. 61 (1993), no. 203, 415-440, S17-S23. MR 1195435 (94b:11085)

16. Carl Ludwig Siegel, Contributions to the theory of the Dirichlet L-series and the Epstein zeta-functions, Ann. of Math. (2) 44 (1943), 143-172. MR 0007760 (4,189c)

17. E. C. Titchmarsh, The theory of the Riemann zeta-function, second ed., The Clarendon Press Oxford University Press, New York, 1986, Edited and with a preface by D. R. Heath-Brown. MR 882550 (88c:11049)

18. A. M. Turing, A method for the calculation of the zeta-function, Proc. London Math. Soc. (2) 48 (1943), 180-197. MR 0009612 (5,173a)

Department of Mathematics, The Ohio State University, 231 West 18th Ave, ColumBUS, OH 43210 